\title{
Insulin receptor substrate-1 gene mutations in NIDDM; implications for the study of polygenic disease
}

\author{
G. A.Hitman ${ }^{1}$, K.Hawrami ${ }^{1}$, M. I.McCarthy ${ }^{1}$, M. Viswanathan ${ }^{2}$, C. Snehalatha ${ }^{2}$, A. Ramachandran ${ }^{2}$, J. Tuomilehto ${ }^{3}$, \\ E. Tuomilehto-Wolf ${ }^{3}$, A. Nissinen ${ }^{3}$, O. Pedersen ${ }^{4}$ \\ ${ }^{1}$ Medical Unit, The Royal London Hospital, Whitechapel, London, UK \\ ${ }^{2}$ Diabetes Research Centre, Madras, India \\ ${ }^{3}$ Department of Epidemiology, National Public Health Institute, Helsinki, Finland \\ ${ }^{4}$ Steno Diabetes Centre, Copenhagen, Denmark
}

\begin{abstract}
Summary Variations in the coding regions of the insulin receptor substrate-1 (IRS-1) gene have recently been suggested to contribute to the susceptibility of non-insulin-dependent diabetes mellitus (NIDDM). The purpose of this study was to examine the role of the IRS-1 missense mutations at codons 972 (glycine to arginine) and 513 (alanine to proline) in two diverse populations from South India and Finland at high risk for NIDDM. DNA was amplified and digested with restriction enzymes BstN1 to detect the codon 972 mutation and Dra III to detect the codon 513 mutation. The codon 513 mutation was not found in the study subjects. The codon 972 mutation was present in $10.3 \%$ of 126 middle-aged NIDDM subjects and $5.3 \%$ of 95 matched control subjects in the South Indians $(p=0.17)$. In elderly Finnish subjects the frequency of the mutation was $7.5 \%$ in 40 NIDDM subjects and $7 \%$ in 42 matched control
\end{abstract}

subjects. The frequency of codon 972 mutation in the South Indian NIDDM subjects was very similar to the two previously published studies in Danish and French subjects although each study individually fails to reach conventional levels of significance. The data from all four ethnic groups were analysed together after ascertaining that significant heterogeneity did not exist between the studies. Overall, the frequency of the codon 972 mutation is found in $10.7 \%$ NIDDM subjects and $5.8 \%$ control subjects ( $p=$ 0.02 ). These studies suggest that the codon 972 mutation of the IRS-1 gene might act as a susceptibility gene predisposing to NIDDM in certain ethnic groups. [Diabetologia (1995) 38: 481-486]

Key words Insulin receptor substrate-1, gene mutations, non-insulin-dependent diabetes mellitus.
Despite intensive investigation few candidate gene mutations have been identified that have a definite role in non-insulin-dependent diabetes mellitus (NIDDM). Approximately $5 \%$ of cases are associated with mutations of the glucokinase gene, insulin receptor gene, insulin gene and mitochondrial DNA [13]. Another subset of NIDDM subjects demonstrate

Received: 14 June 1994 and in revised form: 19 October 1994

Corresponding author: Dr. G. A.Hitman, Medical Unit, The Royal London Hospital, Whitechapel, London, E1 1BB, UK Abbreviations: IRS-1, Insulin receptor substrate-1; NIDDM, non-insulin-dependent diabetes mellitus; OGTT, oral glucose tolerance test; SD, standard deviation; BMI, body mass index; LRCS, likelihood ratio chi square; HOMA, homeostatic model of assessment; PI, phosphatidylinositol. similarities with insulin-dependent diabetes with HLA associations $[4,5]$.

Many groups have shown that insulin resistance is one of the earliest features of NIDDM, preceding detectable abnormalities of insulin secretion [6]. The degree of insulin resistance varies in different ethnic groups; patients with NIDDM from the Indian subcontinent have been found to be more insulin-resistant compared to Europids [7-9]. In longitudinal studies the first metabolic abnormality in the Pima Indians is insulin resistance and there is at least one insulin resistance gene located to chromosome 4, possibly fatty acid binding protein 2 gene or a closelylinked locus $[10,11]$.

The insulin receptor substrate-1 (IRS-1) gene is a plausible candidate locus for insulin resistance in NIDDM. After insulin binds to the $\alpha$ subunit of the in- 
Table 1. Clinical characteristics of the study subjects

\begin{tabular}{lllll}
\hline & $n$ & $\begin{array}{l}\text { Age } \\
\text { (years) }\end{array}$ & $\begin{array}{l}\text { BMI } \\
\left(\mathrm{kg} / \mathrm{m}^{2}\right)\end{array}$ & $\begin{array}{l}\text { Age of on- } \\
\text { set (years) }\end{array}$ \\
\hline $\begin{array}{l}\text { South Indians } \\
\text { Control subjects }\end{array}$ & 58 & $39.7(13.5)$ & $24.3(2.7)$ & - \\
Proband group & 61 & $36.5(6.7)$ & $25.2(3.6)$ & $32.9(6.3)$ \\
Clinic group & 65 & $51.8(10.4)$ & $25.1(3.6)$ & $44.9(9.5)$ \\
Finns & & & & \\
Control subjects & 42 & $75.8(4.0)$ & $25.5(4.6)$ & \\
NIDDM & 40 & $77.2(4.5)$ & $27.2(3.4)$ & \\
\hline
\end{tabular}

Data given as mean \pm (SD).

${ }^{a}$ Full clinical details were only available for 58 of 95 control subjects, the rest being blood donors without a personal or family history of diabetes

sulin receptor, the tyrosine kinase of the $\beta$ subunit undergoes autophosphorylation. This in turn phosphorylates other endogenous protein substrates $[12$, 13]. The first direct substrate for the insulin receptor kinase is IRS-1 which is a hydrophilic phosphoprotein that contains 20 potential tyrosine phosphorylation sites [14]. Six of the tyrosine residues are found in Tyr-Met-X-Met motifs which bind with proteins containing src-homology-2 (SH2) domains. Tyr-Met-XMet motifs in IRS-1 undergo tyrosine phosphorylation after insulin stimulation and bind with several kinases including phosphatidylinositol 3'-kinase (PI $3^{\prime}$ kinase) [15]. PI $3^{\prime}$ kinase plays an important role in the regulation of cell growth [16] and is composed of two subunits - a $110 \mathrm{kDa}$ catalytic subunit and an $85 \mathrm{kDa}$ regulatory subunit which contain $\mathrm{SH} 2$ domains that mediate protein - protein interactions by binding to phosphotyrosine residues in various proteins [17]. The PI $3^{\prime}$ kinase is activated when the SH2 domains of the regulatory subunit bind to phosphorylated Tyr-Met-X-Met motifs in IRS-1. The binding of IRS-1/PI $3^{\prime}$ kinase complexes to the insulin receptor may play a critical role in transmission of insulin signal [18]. IRS-1 also undergoes phosphorylation after activation of the insulin-like growth factor- 1 receptor kinase [19]. Recently in Danish subjects two mutations of the IRS-1 gene were found to be increased in NIDDM compared to control subjects, although each variant by itself was not significantly different from the frequency in control subjects [20]. Mutations were described in codon 972 (a heterozygous missense mutation with a predicted amino acid change of glycine to arginine) present in $11.6 \%$ of NIDDM and $3.9 \%$ of control subjects and in codon 513 (a heterozygous missense mutation with a predicted change of alanine to proline) present in $7 \%$ NIDDM subjects and $2.6 \%$ of control subjects.

The purpose of this study was to study the role of IRS-1 mutations in two diverse populations at high risk of NIDDM and thereby to identify the contribution of these mutations in other ethnic groups. Within the South Indian subjects, NIDDM probands of
61 nuclear families were studied allowing the remainder of the family to be studied if mutations were identified.

\section{Subject and methods}

\section{South Indian subjects}

Population and family study. All diabetic patients were Dravidian (South Indian) subjects recruited via the Diabetic Research Centre of the MV Hospital for Diabetes in Madras. NIDDM was defined by an insidious onset of the disease; if the patient was receiving insulin, this treatment had not been initiated within 5 years of the diagnosis. No subject was insulin-dependent or suffered an episode of diabetic ketoacidosis. The NIDDM patients were derived from two groups: (a) 65 unrelated subjects recruited from the clinic (clinic group) and (b) 61 unrelated probands from nuclear families (proband group) recruited for segregation analysis as previously described [21]. All available non-diabetic family members were tested with an oral glucose tolerance test (OGTT) and diabetic, impaired and normal glucose tolerance were defined by World Health Organization criteria. Paternity was verified by typing at a number of polymorphic markers (HLA-DQA1, tyrosine hydroxylase and glucokinase).

Ninety-five unrelated Dravidian control subjects without personal or first-degree family history of diabetes were recruited from among blood donors $(n=37)$ of from staff and spouses of patients at the MV Hospital for Diabetes $(n=58)$. All the latter subjects had random blood glucose estimation which were all below $6.7 \mathrm{mmol} / \mathrm{l}$. Characteristics of the different groups are given in Table 1 . All studies were approved by the ethical committee of the MV Hospital for Diabetes, Madras, and subjects gave informed consent before venipuncture.

Finnish subjects. The Finnish subjects represent survivors of a population-based cohort of men born in two circumscribed areas of Finland between 1900 and 1919 and recruited into the Seven Countries study (a prospective study of cardiovascular mortality) from 1959 onwards [22]. Cohort members were tested for glucose tolerance for the first time in 1984 and again in 1989 (by $75 \mathrm{~g}$ OGTT) [22]. The survey procedures have been described elsewhere in detail [23]. As correspondence between the results of the two OGTT's was not exact, a composite classification based on the results of both tests was derived. Normal glucose tolerance was defined as a normal OGTT on both occasions in the absence of hypoglycaemic medication and diabetic glucose tolerance was assigned where an individual attained World Health Organisation criteria for diabetes at either OGTT or was receiving anti-diabetic drugs [5]. The mean (SD) age of all subjects at the 1989 test was 76.6 (4.4) years and the BMI $26.2(3.6) \mathrm{kg} / \mathrm{m}^{2}$ : the two subject groups (diabetic and normal) did not differ significantly for these parameters (Table 1 ). In the current study, analysis was performed in a random selection of diabetic $(n=40)$ and nondiabetic subjects $(n=42$; Table 1$)$.

Laboratory methods. The coding regions of IRS-1 were amplified from either genomic DNA or crude lysates using primers which encompass the codon 972 and 513 variants, respectively. The experimental conditions were those as previously described [20]. The primers used were for Codon 513:

Forward primer $19535^{\prime}$ gcg gtg agg agg agc taa gc 3' 1972

Reverse primer $22003^{\prime}$ ggg cag ggt cag gag tca ccg 5' 2220

and for Codon 972: 
Table 2. IRS-1 Codon 972 mutation; frequency in the present and published studies

\begin{tabular}{|c|c|c|c|c|}
\hline \multirow[t]{2}{*}{ Ethnic group } & \multicolumn{4}{|c|}{ Frequency of IRS- 1 codon 972 variant } \\
\hline & NIDDM & $\begin{array}{l}\text { Control } \\
\text { subjects }\end{array}$ & $\begin{array}{l}\text { LRCS } \\
\S\end{array}$ & $p \S$ \\
\hline Sou & $13 / 126$ & $5 /$ & 1 & 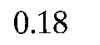 \\
\hline Finr & $3 / 40(7.5 \%)$ & $3 / 4$ & 0.04 & 0.84 \\
\hline Finnish $[27]^{\mathrm{a}}$ & $11 / 112(9.8 \%)$ & $9 / 104(8.7 \%)$ & 0.09 & 0.73 \\
\hline Danish $[20]^{\mathrm{a}}$ & $10 / 86(11.6 \%)$ & $3 / 76(3.9 \%)$ & 3.42 & 0.06 \\
\hline French $[29]^{\mathrm{a}}$ & $26 / 233(11.2 \%)$ & $9 / 130(6.9 \%)$ & 1.80 & 0.17 \\
\hline Combined & $63 / 597(10.6 \%)$ & $29 / 447(6.5 \%)$ & & $0.026^{\mathrm{b}}$ \\
\hline
\end{tabular}

${ }^{a}$ Reference number from which data has been taken. ${ }^{\mathrm{b}}$ Homogeneity test for the four studies $p=0.75$ ) i.e. no significant hetereogeneity between the studies). Overall odds ratio (Mantel-Haenszel) $1.7 ; 95 \%$ confidence limits $1.1-2.7 ; p=$ 0.026 . $^{\mathrm{c}}$ LRCS, Likelihood ratio chi square; the previously published data sets were recalculated using the LRCS and the $p$ values calculated by exact permutational methods

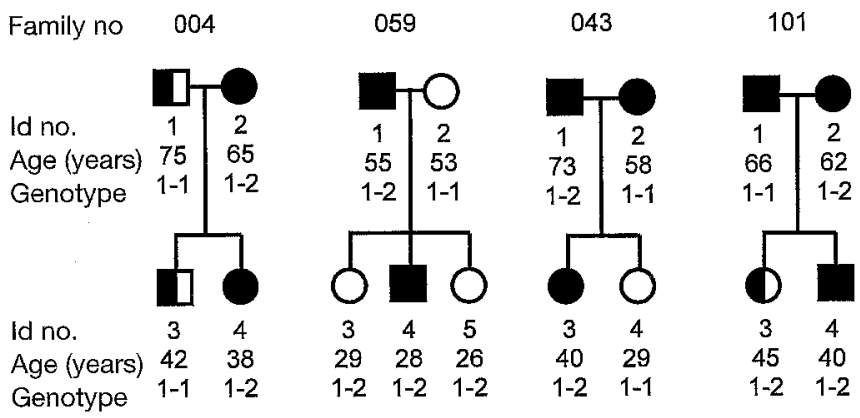

KEY $\quad \square_{\text {NIDDM }} \square_{\text {NGT }} \square_{\text {IGT }}$

Fig. 1. South Indian pedigrees in which the NIDDM proband possessed the IRS-1 codon 972 variant. id. no., identity number; genotype; $1=$ normal sequence at IRS-1 codon 972, $2=$ IRS- 1 codon 972 variant. NGT, normal glucose tolerance test; IGT, impaired glucose tolerance

Forward primer $33395^{\prime} \mathrm{ctt}$ ctg tca ggt gtc cat cc $3^{\prime} 3358$ Reverse primer $35823^{\prime}$ cga tgc acc tgt gga gcg gt 5'3601 Restriction enzyme digestion was carried out using the appropriate restriction enzyme; Bst N1 for the codon 972 mutation, and Dra III for the codon 513 mutation. The fragments were analysed after electrophoresis on a $4.5 \%$ agarose gel and alleles sized by reference to $1 \mathrm{~kb}$ marker (BRL, Paisley, Strathclyde, UK). A positive control for each IRS-1 variant was included in all experiments.

\section{Statistical analysis}

Significance between test groups were tested using the Likelihood ratio statistic (LRCS) using $2 \times 2$ chi square tables. Exact permutational methods (StatXact-Turbo; Cytel Corporation, Cambridge, Mass., USA) were preferred over asymptotic tests in view of the small expected frequencies in some categories. The Homeostatic Model of Assessment (HOMA) measures of beta-cell function and insulin sensitivity were calculated from fasting plasma insulin and glucose levels [24]. Significance between continuous variables and genotype was tested by an analysis of variance (SPSS Windows; release 6). Lod scores were calculated using the LINKAGE package accessed remotely at the Human Genome Mapping Project Resource Centre, Harrow, UK. Parameters for the models used were derived from previous segregation analysis in the South Indians consisting of autosomal dominant and oligogenic models [21].

\section{Results}

513 mutations. Two patterns were observed after DNA was digested with Dra III; wild-type gene, one band sized $286 \mathrm{bp}$ and in a sample from a subject who was heterozygous for the mutation three bands sized 268, 168 and $100 \mathrm{bp}$. The codon 513 mutation was not found in any of the study subjects. The presence of a positive control in each gel confirmed our potential to detect the mutation under the experimental conditions used.

\section{2 mutation}

\section{(i) South Indian subjects}

Two patterns were observed after DNA was digested with Bst NI; the wild-type gene 3 bands sized 23, 81 and $158 \mathrm{bp}$ whilst a sample from a subject who was heterozygous for the mutation was characterised by 5 bands sized 23, 81, 158, 107 and 51 bp. The codon 972 mutation was identified in 9 out of 65 $(13.8 \%)$ diabetic subjects in the clinic group and 4 out of $61(6.6 \%)$ of the NIDDM probands (total diabetic subjects $13 / 126 ; 10.3 \%)$ but only $5 / 95(5.3 \%)$ control subjects (Table 2). This did not reach significance at the $5 \%$ level LRCS $\left(\chi^{2}=1.9\right.$; exact $p=0.18$ ). Although the frequency of the 972 mutation was increased in the clinic group compared to the proband group, it was not statistically significant $(p=0.2)$. The frequency of the mutation in the clinic group compared to control subjects just missed conventional levels of significance $(p=0.06)$. No differences were found between NIDDM subjects (clinic group plus proband group) with or without the mutation for body mass index, treatment, age of onset of NIDDM or cholesterol and triglycerides at diagnosis. The family trees of the four probands who possessed the codon 972 mutation are presented in Figure 1. In all families, at least one diabetic parent possessed the mutation. The mutation was also present in siblings with normal glucose tolerance (family 059; id no 3 and 5) and impaired glucose tolerance (family 101; id no 3 ). Lod scores for linkage between NIDDM and the 972 mutation were calculated for a variety of genetic models assuming a zero recombination fraction and confirmed the limited information content of these particular families which is apparent on inspection. The maximum (positive or negative) lod score obtained 
was 0.23 for family 004 under a dominant model of inheritance.

\section{(ii) Finnish subjects}

The codon 972 mutation was present in equal numbers of NIDDM subjects $(7.5 \%)$ and matched control subjects ( $7.0 \%$; Table 2). Fasting serum insulin levels in the three control subjects heterozygous for the 972 mutation were 8,9 and $19 \mu \mathrm{U} / \mathrm{ml}$ : these did not differ from the values obtained in the other control subjects (geometric mean, SD range: 6.5, 2.616.5). In addition, there were no differences between individuals with and without the mutation for 2-h serum insulin levels, or for HOMA measures of beta-cell function and insulin sensitivity or BMI (control $[n=3]$ and diabetic $[n=3]$ subjects analysed separately).

\section{Discussion}

The study of the genetic determinants of NIDDM is complicated by the likelihood that NIDDM is a heterogeneous disease both at the genotypic and phenotypic levels. Insulin resistance is a prominent feature of NIDDM in South Indians and therefore this population is a good test for candidate genes associated with insulin resistance. In contrast, the elderly Finnish men have all survived the possibility of premature ischaemic heart disease. Ischaemic heart disease is thought to cluster with NIDDM, dyslipidaemia and central obesity and is often characterised by insulin resistance; this aggregation has been called 'syndrome $X$ ' or the 'insulin resistance syndrome' [25]. Diabetes in the elderly Finnish men is likely therefore to represent 'non-syndrome $X$ ' disease (e.g. the aetiology is likely to be due to a predominant betacell defect). Indeed, a strong association has been found in this group between NIDDM and HLA and a weaker association with the glucokinase gene [3, 26]. The possibility of a predominant beta-cell defect in the elderly Finns may explain the absence of an increased frequency of the IRS-1 variant 972 in that patient population; however, the number of subjects studied is relatively small and it does not exclude the possibility of other IRS-1 variants. This has been largely overcome by a recent study of middle-aged NIDDM subjects from Finland in whom both diabetic patients and control subjects were found to have very similar prevalence of the codon 972 variant as in our studies [27]. Although a number of other IRS1 variants were found in this study, none accounted for more than $2.7 \%$ of the diabetic subjects.

The results in the South Indians are more difficult to interpret; the codon 972 mutation was found twice as frequently in the diabetic patients compared to control subjects but this did not reach conventional levels of significance in this population alone. South Indian Dravidians are considered on anthropological and genetic grounds to share common ancestry with Europid and other Caucasoid groups [28]. Since the frequency of the mutation in the South Indian diabetic and control groups is similar to that found in other studies of Europid groups [20, 27, 29] it is instructive to estimate the number of subjects necessary to achieve conventional levels of significance, assuming the codon 972 mutation is present in $10 \%$ of diabetic subjects and $5 \%$ of non-diabetic subjects using exact permutational methods. To detect an association with a $p$ value of $0.01,380$ diabetic patients and 380 control subjects would need to be studied and for a $p$ value of $0.05,220$ subjects would be required in each study group. Therefore, all the published studies individually lack the power to detect an association of this magnitude between NIDDM and the codon 972 IRS-1 mutation. When the data from all these studies is combined an overall association between NIDDM and the IRS-1 codon 972 variant clearly emerges. Before data from the four ethnic groups were combined a homogeneity test (Zelen Statistic) confirmed the validity of the analysis $(p=0.75)$. The overall odds ratio (OR; Mantel-Haenszel) was significant $(\mathrm{OR}=1.7 ; 95 \%$ confidence limits $1.1-2.7$; the exact $p$ value $=0.026$ ) the mutation being present in $10.6 \%$ of NIDDM subjects and $6.5 \%$ of control subjects. The combined analysis would therefore suggest that the IRS-1 codon 972 missense mutation (glycine to arginine) might act as a gene variant in the predisposition for NIDDM in several ethnic groups.

The codon 972 variant was present in about $5 \%$ of South Indian control subjects. This may be explained by the admixture of subjects destined to develop diabetes within our control groups. However, the frequency of this variant was similar (3.9 and $7.0 \%$ ) in the Danish and French control groups which were better characterised for glucose tolerance.

Recent segregation analysis using the computerised programme COMDS has indicated that the inheritance of diabetes in the South Indians can be explained by a combination of a gene contributing $40 \%$ to diabetes and the rest being explained by a polygenic effect affecting the range of glucose tolerance [21]. The IRS-1 gene variant is clearly not acting as a major gene for NIDDM, but rather is likely to be a susceptibility locus, which is itself neither necessary nor sufficient for the development of NIDDM and is contributing to the polygenic effect. This effect can be seen by inspection of the pedigrees in which the proband possessed the IRS-1 codon 972 variant (Fig. 1). In all families the proband had an affected parent who also carried the variant; however, two siblings who also carried the variant had normal glucose tolerance (family 059; id no 3 and 5) and one 
had impaired glucose tolerance (family 101; id no 3) suggesting that this variant by itself does not necessarily lead to NIDDM at least in early life.

These results raise the problem of assessing the relevance of mutations/variants identified in candidate genes for NIDDM; particularly when these are infrequent and seen in both diabetic patients and control subjects. Demonstration of an excess frequency of the mutation in cases compared to control subjects, especially if reproduced in a number of populations is clearly supportive that the mutation contributes to disease pathogenesis. However, the effect of the mutation needs to be proven at the cellular level by expression studies since until the variant is proven to be of physiological significance, the associations described could still reflect linkage disequilibrium with another mutation in that gene (in this case IRS-1) or in a closely linked gene. The detection of susceptibility loci which only contribute a small proportion of disease pathogenesis may be best achieved by using well-constructed large population association studies. In this type of study the diabetic and control subjects should come from the same sampling frame to minimise the chances of hidden population stratification between the test subjects. Whilst it is comparatively easy to identify subjects with NIDDM, it is far from clear how to define a control subject since the distribution of glucose tolerance is not a bimodal distribution and the reproducibility of the OGTT is relatively poor within an individual [30]. If a large enough population is available, the best non-diabetic control subject might be an individual with a glucose tolerance in the lowest decile of that population group; ideally the normal glucose status would be confirmed on repeat testing. Population association studies could also be used to study genes contributing individual traits leading to NIDDM, rather than to NIDDM itself. Thus, if the IRS-1 codon 972 mutation has a direct effect on insulin sensitivity, normoglycaemic subjects with and without the mutation could be studied for parameters of insulin resistance.

An alternative strategy which overcomes the problems of hidden population stratifications in population association studies is to use methods based on the haplotype relative risk [31]. In these experiments nuclear families with one affected offspring are used to test for deviations in linkage equilibrium. The 'cases' are made up of the alleles transmitted to the affected offspring and the 'control' alleles made up of the alleles not transmitted from the parent to the child. The statistical comparison is then between the transmitted and the non-transmitted alleles. If sufficient nuclear pedigrees can be obtained this may be the method of choice to demonstrate linkage disequilibrium. The problem with this method is that identification of nuclear pedigrees is rendered difficult in a disease which is characterised by a late age of onset and premature mortality. Co-segregation of the mu- tation with the disease in affected family members is the classic means of proving linkage between the mutation and disease. However, linkage analysis lacks the power to detect susceptibility loci (such as IRS-1 is likely to be) which are neither necessary nor sufficient for disease expression [32]. If NIDDM turns out to be a heterogenous disease, and especially if it has a significant polygenic component, these problems need to be addressed if we are going to make progress in identifying risk genes in this disease.

Acknowledgements. Grants from Wellcome Trust, Medical Research Training Fellowship (Mark McCarthy), Velux Foundation and Danish Diabetes Association.

\section{References}

1. Hattersley AT, Turner RC (1993) Mutations of the glucokinase gene and type 2 diabetes. Quart J Med 86: 227-232

2. Taylor SI (1992) Molecular mechanisms of insulin resistance, lessons from patients with mutations in the insulinreceptor gene. Diabetes 41: 1473-1490

3. Gerbitz K-D (1992) Does the mitochondrial DNA play a role in the pathogenesis of diabetes? Diabetologia 35: 1181-1186

4. Rich SS, French LR, Sprafka JM, Clements JP, Goetz FC (1993) HLA-associated susceptibility to type 2 (non-insulin-dependent) diabetes mellitus: the Wadena City Health study. Diabetologia 36: 234-238

5. Tuomilehto-Wolf E, Tuomilehto J, Hitman G et al. (1993) Genetic susceptibility to non-insulin-dependent diabetes mellitus and glucose intolerance are located in the HLA region. BMJ 307: 155-159

6. Martin BC, Warram JH, Krolewski AS, Bergman RN, Soeldner JS, Kahn CR (1992) Role of glucose and insulin resistance in development of type 2 diabetes mellitus: results of a 25 year follow-up study. Lancet 340: 925-929

7. Mohan V, Sharp PS, Aber VR, Mather KM, Kohner EM (1987) Insulin resistance in maturity onset diabetes of the young. Diabetes and Metabolism 13: 193-197

8. Sharp PS, Mohan V, Levy JC, Mather KM, Kohner EM (1987) Insulin resistance in patients of Asian, Indian and European origin with non-insulin dependent diabetes. Horm Metab Research 19: 84-85

9. Gelding SV, Niththyndanthan R, Chan SP et al. (1994) Insulin sensitivity in non-diabetic relatives of patients with non-insulin-dependent diabetes from two ethnic groups. Clin End 40: 55-62

10. Lillioja S, Mott DM, Spraul M et al. (1993) Insulin resistance and insulin secretory dysfunction as precursors of non-insulin-dependent diabetes mellitus. N Engl J Med 329: 1988-1992

11. Prochazka M, Lillioja S, Tait JF et al. (1993) Linkage of chromosomal markers on $4 \mathrm{q}$ with a putative gene determining maximal insulin action in Pima Indians. Diabetes 42: 514-519

12. Rosen OM (1987) After insulin binds. Science 237: 14521458

13. Khan CR, White MF (1988) The insulin receptors and the molecular mechanism of insulin action. J Clin Invest 82 : $1151-1156$

14. Sun XJ, Rothenberg P, Khan CR et al. (1991) Structure of insulin receptor kinase substrate IRS-1 defines a unique signal transduction protein. Nature 352: 73-77 
15. Sholeson SE, Chatterjee S, Chaudhuri M, White MF (1993) YMXM motifs of IRS-1 define substrate specifity of the insulin receptor kinase. Proc Natl Acad Sci USA 268: 8204 8212

16. Cantly LC, Auger KR, Carpenter C, Duckworth B, Kapellar R, Soltoff I (1991) Oncogenes and signal transduction. Cell 64: 281-302

17. Mayers MG, Sun XJ, Cheatam B et al. (1993) Association of IRS-1 is a common element in insulin and insulin like growth factor 1 signalling to the phosphatidylinositol 3 '-kinase. Endocrinology 132: 1421-1430

18. Backer JM, Mayer MG, Sun XJ et al. (1993) Association of IRS-1 with the insulin receptor and the phosphatidylinositol 3'-kinase. J Biol Chem 268: 8204-8212

19. Mayers MJ, White MF (1993) The new elements of insulin signalling. Diabetes 42: 643-650

20. Almind K, Bjørbaek C, Vestergaard H, Hansen T, Echwald $S$, Pederson O (1993) Amino acid polymorphisms of insulin receptor substrate-1 in non-insulin dependent diabetes mellitus. Lancet 342: 828-832

21. McCarthy MI, Hitman GA, Shields DC et al. (1994) Family studies of non-insulin-dependent diabetes mellitus in South Indians. Diabetologia 37: 1221-1230

22. Stengård JH, Tuomilehto J, Pekkanen J et al. (1992) Diabetes mellitus, impaired glucose tolerance and mortality among elderly men: the Finnish cohorts of The Seven Countries Study. Diabetologia 35: 760-765

23. Tuomilehto J, Nissinen A, Kivelä S-L et al. (1986) Prevalence of diabetes mellitus in elderly men aged 65 to 84 years in eastern and western Finland. Diabetologia 29: 611-615
24. Matthews D, Hosker JP, Rodenski A, Naylor BA, Treacher DF, Turner RC (1985) Homeostasis model assessment: insulin resistance and $\mathrm{B}$-cell function from fasting plasma glucose and insulin concentrations in man. Diabetologia 28: $412-419$

25. Reaven G (1988) Role of insulin resistance in human disease. Diabetes 37: 1595-1607

26. McCarthy MI, Hitman GA, Hitchins M et al. (1994) Glucokinase gene polymorphisms: a genetic marker for glucose intolerance in a cohort of elderly Finnish men. Diabet Med 11: 198-204

27. Laakso M, Malkki M, Kekalainen P, Kuusisto J, Deeb SS (1994) Insulin receptor substrate-1 variants in non-insulindependent diabetes. J Clin Invest 94: 1141-1146

28. Cavalli-Sforza LL, Pieza A, Menozzi P, Mountain J (1988) Reconstruction of human evolution: bringing together genetic, archeological, and linguistic data. Proc Natl Acad Sci USA 85: 6002-6006

29. Hager J, Zouali H, Velho G, Froguel P (1993) Insulin receptor substrate (IRS-1) gene polymorphisms in French NIDDM families. Lancet 342: 1430

30. Home P (1988) The OGTT: gold that does not shine. Diabet Med 5: 313-314

31. Terwilliger J, Ott J (1992) A haplotype-based 'haplotype relative risk' approach to detecting alleleic associations. Hum Hered 42: 337-346

32. Greenberg DA (1993) Linkage analysis of "necessary" disease loci versus "susceptibility" loci. Am J Hum Genet 52: 135-143 